\section{(2) OPEN ACCESS}

\title{
Ethnic disparities in preventable hospitalisation in England: an analysis of 916375 emergency admissions
}

\author{
Jakob Petersen $\odot,{ }^{1}$ Jens Kandt, ${ }^{2}$ Paul Longley ${ }^{1}$
}

\begin{abstract}
- Additional online supplemental material is published online only. To view, please visit the journal online (http://dx.doi.org/10.1136/ jech-2020-216005).

${ }^{1}$ Geography, UCL, London, UK ${ }^{2}$ The Bartlett Centre for Advanced Spatial Analysis (CASA), University College London, London, UK
\end{abstract}

\section{Correspondence to}

Dr Jakob Petersen, Geography, UCL, London WC1E 6BT, UK; jakob.petersen1@Ishtm.ac.uk

Received 8 November 2020 Accepted 14 June 2021 Published Online First 24 June 2021

\section{Check for updates}

(C) Author(s) (or their employer(s)) 2022. Re-use permitted under CC BY. Published by BMJ.

To cite: Petersen J, Kandt J, Longley P. J Epidemiol Community Health 2022;76:85-91.

\section{ABSTRACT}

Objectives To study ethnic inequalities in ambulatory care sensitive conditions (ACSC) in England.

Design Observational study of inpatient hospital admission database enhanced with ethnicity coding of patient surnames. The primary diagnosis of the first episode in spells with emergency admission were coded with definitions for acute ACSC, chronic ACSC and vaccine-preventable diseases.

Setting National Health Service England.

Participants 916375 ACSC emergency admissions in 739618 patients were identified between April 2011 and March 2012.

Main outcome measures ORs of ACSC for each ethnic group relative to the White British majority group adjusted for age, sex and area deprivation.

Results Acute ACSC admission risk adjusted for age and sex was particularly high among Other (OR 1.73; $95 \% \mathrm{Cl} 1.69$ to 1.77$)$ and Pakistani $(1.51 ; 95 \% \mathrm{Cl}$ 1.48 to 1.54$)$ compared with White British patients. For chronic ACSC, high risk was found among Other (2.02; $95 \% \mathrm{Cl} 1.97$ to 2.08$)$, Pakistani $(2.07 ; 95 \% \mathrm{Cl} 2.02$ to 2.12) and Bangladeshi (1.36; $95 \% \mathrm{Cl} 1.30$ to 1.42 ). For vaccine-preventable diseases, other $(2.42 ; 95 \% \mathrm{Cl}$ 2.31 to 2.54$)$, Pakistani $(1.94 ; 95 \% \mathrm{Cl} 1.85$ to 2.04$)$, Bangladeshi (1.48; $95 \% \mathrm{Cl} 1.36$ to 1.62), Black African $(1.45 ; 95 \% \mathrm{Cl} 1.36$ to 1.54$)$ and white other $(1.38 ; 95 \%$ Cl 1.33 to 1.43) groups. Elevated risk was only partly explained in analyses also adjusting for area deprivation. Conclusions ACSC admission was especially high among individuals of Bangladeshi, Pakistani, Black African, white other or other background with up to twofold differences compared with the white British group. This suggests that these ethnic groups are not receiving optimal primary care.

\section{INTRODUCTION}

Emergency hospital admissions are distressing for patients, associated with poorer long-term outcomes and are costly to the healthcare system. Many healthcare systems are therefore undergoing reforms to reduce emergency admissions by improving early detection, treatment and monitoring of a range of conditions that could be treated or prevented in less intensive settings, that is, primary and community care services. ${ }^{12}$ These conditions are known as ambulatory care sensitive conditions (ACSCs). ACSCs include acute, chronic and vaccine-preventable conditions such as urinary tract infections, chronic obstructive pulmonary disease (COPD) and pneumonia. ACSC admissions have been associated with patients under the age 5 years, the elderly, deprivation and ethnicity. ${ }^{1}$

The English National Health Service (NHS) saw a 40\% rise in ACSC admissions between 2001 and $2011^{1}$ and a $42 \%$ rise in emergency admissions between 2006 and 2017 making this a policy area of urgency. ${ }^{3}$ ACSC indicators were introduced into the NHS Commissioning Outcome Framework in 2012 to monitor this area for quality of care improvements for the general population. ${ }^{1}$ While ACSC has been studied before in England, there has to our knowledge not been a study of ethnic inequalities in ACSC in England nor of its geographical distribution for these groups. A study of ACSC is particularly pertinent for the understanding of ethnic inequalities, because they are indicative of how patients from different minorities access and navigate the healthcare system. Studies in USA, New Zealand and Scotland have found higher risk of ACSC admission for many ethnic minorities compared with the white majority populations. ${ }^{4-6} \mathrm{~A}$ recent Scottish study found that South Asian groups had higher risk of ACSC admission compared with the white majority group. ${ }^{4}$

The primary objective of this study was to elucidate whether ethnicity plays an important role in ACSC among emergency admissions in England at a national and regional level. For this study, we gathered data on hospital admission from Hospital Episode Statistics (HES) for different ethnic groups in 2011 and linked them to the 2011 Census population estimates.

The completeness of ethnicity data in HES was very low in the $1990 \mathrm{~s}$, but has since improved. ${ }^{7}$ To address potential gaps in the ethnicity records, a freely available software, Ethnicity Estimator (EE) was used to code patient surnames to major ethnic groups. ${ }^{8}$ We report on the EE as a secondary objective of this paper.

\section{METHODS}

Inpatient hospital admission records with an emergency admission route were obtained from NHS England's HES, April 2011-March 2012. Diagnoses in HES are coded to the Tenth Revision of the International Classification of Diseases system. ${ }^{9}$ The primary diagnosis of the first episode in spells with emergency admission were coded with definitions for acute ACSC, chronic ACSC and vaccinepreventable diseases ${ }^{1}$ (online supplemental table S1), for definitions). Only the first episode for each admission was considered to focus on whether the 
admission could have been prevented. The data were deduplicated so that a patient could only contribute to the risk of each ACSC group once.

In this population-based study, the denominators came from the 2011 Census and the numerators from HES. The census ethnicity classification is the result of extensive consultation ${ }^{10}$ and HES ethnicity classification was based on the 2001 Census. ${ }^{11}$ We made a few modifications to harmonise the differences between how data are collected and released at small area level for the 2001 and 2011 censuses. HES-recorded ethnicity categories for black other and other were combined accordingly and all mixed groups were analysed as one. For Census base population data, Arabic and black other were combined with other.

There is a growing literature about the use of names to impute ethnicity in studies where this information is not routinely collected or is available through data linkage. ${ }^{812-14}$ The Onomap software developed at University College London ${ }^{815}$ has been used in over 60 scientific studies and social equity audits in applications as diverse as accident and emergency department utilisation, residential segregation, labour market discrimination and the composition of company boards. Building on Onomap, the EE software deployed in this study was developed in collaboration with the Office for National Statistics to optimise imputation of ethnic minority categories in the context of England and Wales. We use the optimised EE software to capture missing self-reported ethnicity information in HES across a full range of ethnic groups. ${ }^{81213}$ To retain full anonymity, the surname coding was carried out in an air-gapped, secure data facility by NHS Digital linking name information in the Patient Demographic Service to HES. We calculated the proportion of each ethnic group correctly predicted by the EE software as a secondary outcome.

The census denominator data were obtained at local authority level. Area deprivation quintiles were accordingly coded at this level ${ }^{16}$ by creating population-weighted mean scores and quintiles. ORs were calculated for each ethnic group using logistic regression with White British as reference group adjusting for age, sex, and area deprivation. Standardised morbidity ratio (SMR) was calculated by ethnic group and local authority district for a combined ACSC outcome (acute, chronic or vaccinepreventable). The results for areas with less than 20 cases were suppressed. All analyses were carried out using Stata V.14 ${ }^{17}$ and maps were created using QGIS V.3.16. ${ }^{18}$

\section{RESULTS}

A total of 916375 ACSC emergency admissions in 739618 patients were identified in 2011/12. The most common ACSCs were urinary tract infections $(17.0 \%$ of all ACSC), COPD $(12.5 \%)$, and pneumonia $(10.5 \%)$ (table 1$)$. The majority of ACSC patients were from the white British group (83.3\%), followed by white other (3.7\%), Pakistani (2.5\%), other (2.2\%), Indian (2.0\%), Black African (1.1\%), Asian other (1.1\%), White Irish (1.1\%), mixed (1.0\%), Black Caribbean (0.9\%), Bangladeshi $(0.6 \%)$ and Chinese $(0.2 \%)$ (table 2$)$. Among all patients, especially those from other and Pakistani groups had a significantly higher risk of emergency admission for preventable causes with some variation between gender and whether acute ACSC, chronic ACSC or vaccine-preventable diseases.

Age-standardised and sex-standardised ORs of acute ACSC admissions were particularly high for other (OR 1.73; 95\% CI 1.69 to 1.77$)$ and Pakistani $(1.51 ; 95 \%$ CI 1.48 to 1.54$)$ patients (figure 1). For chronic ACSC, the ORs were very high for other (2.02; $95 \%$ CI 1.97 to 2.07), Pakistani $(2.07 ; 95 \%$ CI 2.02 to
Table 1 Emergency admissions for ambulatory care sensitive conditions in 2011

\begin{tabular}{|c|c|c|}
\hline Conditions & Freq. & $\%$ \\
\hline \multicolumn{3}{|l|}{ Acute ACSC } \\
\hline Cellulitis & 63044 & 6.9 \\
\hline Dehydration & 10615 & 1.2 \\
\hline Dental conditions & 10485 & 1.1 \\
\hline ENT & 84219 & 9.2 \\
\hline Gangrene & 1346 & 0.1 \\
\hline Gastroentereritis & 73403 & 8.0 \\
\hline Nutritional deficiencies & 205 & $<0.1$ \\
\hline Pelvic inflammatory disease & 4805 & 0.5 \\
\hline Perforated ulcer & 4980 & 0.5 \\
\hline UTI/pyelonephritis & 155948 & 17.0 \\
\hline Subtotal & 409050 & 44.5 \\
\hline \multicolumn{3}{|l|}{ Chronic ACSC } \\
\hline Angina & 61625 & 6.7 \\
\hline Asthma & 54613 & 6.0 \\
\hline COPD & 114454 & 12.5 \\
\hline Congestive heart failure & 56448 & 6.2 \\
\hline Convulsion/epilepsy & 77783 & 8.5 \\
\hline Diabetes complications & 23142 & 2.5 \\
\hline Hypertension & 6648 & 0.7 \\
\hline Iron-deficiency anaemia & 12075 & 1.3 \\
\hline Subtotal & 406788 & 44.4 \\
\hline \multicolumn{3}{|l|}{ Vaccine-preventable diseases } \\
\hline Influenza & 1163 & 0.1 \\
\hline Pneumonia & 96525 & 10.5 \\
\hline TB & 1618 & 0.2 \\
\hline Other vaccine preventable & 1231 & 0.1 \\
\hline Subtotal & 100537 & 10.9 \\
\hline Total & 916375 & 100 \\
\hline
\end{tabular}

ACSC, ambulatory care sensitive conditions; COPD, chronic obstructive pulmonary disease; ENT, ear, nose, and throat; TB, tuberculosis; UTI, urinary tract infection.

2.11), and Bangladeshi (1.36; 95\% CI 1.30 to 1.42) groups. For vaccine-preventable diseases, the same was true for other (2.42; 95\% CI 2.31 to 2.54), Pakistani (1.94; 95\% CI 1.85 to 2.04), Bangladeshi $(1.48 ; 95 \%$ CI 1.36 to 1.62$)$, Black African $(1.45 ; 95 \%$ CI 1.36 to 1.54$)$ and white other $(1.38 ; 1.33$ to $1.43)$ groups. For the combined ACSC outcome, two groups had particularly elevated ORs, Pakistani (1.74; 95\% CI 1.71 to 1.76$)$ and other $(1.92 ; 95 \%$ CI 1.88 to 1.95$)$ (table 3$)$. Standardising the incidence for area deprivation showed that the results for the groups with elevated risk was partly, but not entirely explained by area deprivation (figure 1, table 3 ). Similar findings were obtained when analysing the data without surname imputation (online supplemental figure S1 and table S2). Overall, Chinese, mixed and white Irish had risks well below White British and some groups had comparable risks. Especially, the Pakistani and other groups appear to be faced with problems around healthcare access for acute and chronic preventable conditions. The results on vaccine-preventable diseases suggest that many ethnic minorities are less well protected.

The maps of ACSC SMR showed higher risk in Inner London and the old industrial areas of the Midlands and the North (figure 2). This was consistent for both White British and 
Table 2 Patients with ACSC by ethnic group and sensitivity of EE software (Kandt and Longley, 2018) in predicting NHS ethnic group where missing together with the Census 2011 population denominators for England

\begin{tabular}{|c|c|c|c|c|c|c|c|}
\hline & NHS recorded & & $\begin{array}{l}\text { NHS recorded +name } \\
\text { imputation }\end{array}$ & & $\begin{array}{l}\text { Sensitivity of EE } \\
\text { software }\end{array}$ & $\begin{array}{l}\text { Specificity of EE } \\
\text { software }\end{array}$ & $\begin{array}{l}\text { Population } \\
\text { denominator }\end{array}$ \\
\hline Ethnic group & Freq. & $\%$ & Freq. & $\%$ & $\%$ & $\%$ & Freq. \\
\hline Asian other & 7558 & 1.0 & 7967 & 1.1 & 18.7 & 99.5 & 819402 \\
\hline Bangladeshi & 4331 & 0.6 & 4654 & 0.6 & 59.5 & 99.6 & 436514 \\
\hline Chinese & 1601 & 0.2 & 1760 & 0.2 & 68.5 & 99.9 & 379503 \\
\hline Indian & 13859 & 1.9 & 14986 & 2.0 & 72.4 & 99.1 & 1395702 \\
\hline Pakistani & 17203 & 2.3 & 18551 & 2.5 & 79.9 & 98.8 & 1112282 \\
\hline Black African & 7557 & 1.0 & 8329 & 1.1 & 55.9 & 99.3 & 977741 \\
\hline Black Caribbean & 6800 & 0.9 & 6975 & 0.9 & 9.8 & 99.7 & 591016 \\
\hline Other & 15629 & 2.1 & 15948 & 2.2 & 4.3 & 99.6 & 881170 \\
\hline White other & 25053 & 3.4 & 27452 & 3.7 & 42.5 & 97.1 & 2430010 \\
\hline White British & 588333 & 79.5 & 616327 & 83.3 & 90.1 & 61.7 & 42279236 \\
\hline White Irish & 6056 & 0.8 & 7867 & 1.1 & 47.8 & 94.9 & 517001 \\
\hline Mixed & 7280 & 1.0 & 7280 & 1.0 & - & - & 1192879 \\
\hline Missing & 38358 & 5.2 & 1522 & 0.2 & - & - & - \\
\hline Total & 739618 & 100 & 739618 & 100 & - & - & 53012456 \\
\hline
\end{tabular}

EE, ethnicity estimator; NHS, National Health Service.

Pakistani, although Pakistani patients experienced higher risk in other parts of the country.

The sensitivity of the EE software to correctly predict the HESrecorded ethnicity varied by ethnic group, for example, $>90 \%$ for white British and $<10 \%$ for black Caribbean (table 2).

\section{DISCUSSION}

Ethnic inequalities in health are manifested in numerous ways and predominantly have adverse social determinants such as poor living and working conditions, discrimination, social exclusion, adverse health behaviours and poor healthcare accessibility. ${ }^{19}$ The incidence of preventable hospitalisations indicates how patients from ethnic minorities access and navigate the healthcare system. We found that among all emergency admissions, especially patients with Other, Bangladeshi, Pakistani, Black African and white other backgrounds had higher OR of emergency ACSC relative to the White British majority group, for example, close to twice as high for the Pakistani and the other groups.

A study of ACSC in England, 2001-2011, found an overall rise in ACSC admissions of $40 \% .^{1}$ Part of the increase was attributed to the ageing population, although age-standardised rates still increased $25 \%$ over the same time period. The study found the greatest increases in urinary tract infections, pyelonephritis, pneumonia, gastroenteritis and COPD. The authors stated that the first two conditions are difficult to diagnose in the elderly and hence not always detected and treated early enough to prevent a hospital admission. ${ }^{1}$ For pneumonia, they found that cases in the elderly have risen over decades in many countries. They speculated whether the increase in ACSC reflected an ageing population with increasingly complex health and social care needs. They also observed that the rise in ACSC coincided with a rise in emergency admission for non-ACSC. The authors concluded that more reforms were required to improve how primary, secondary, community and adult social care services were organised and delivered under the banner of 'integrated care'.

As evident from this and other papers, several ethnic minorities have been associated with high relative risk of ACSC conditions; notwithstanding that they only account for a smaller proportion of the ACSC problem overall. ${ }^{5}$ A recent Scottish study found that ACSC admissions were significantly higher in South Asian groups compared with the white Scottish population. ${ }^{4}$ US studies have found higher rates among black, Hispanic and other groups relative to the white majority group. ${ }^{6}$ Studies from New Zealand found higher rates among Maori and Pacific Islanders compared with the white majority group. ${ }^{6}$ Common for these studies are calls to improve not only access to primary care but also the quality of care received for both ethnic and migrant groups.

The admission risk was generally lower for Chinese patients compared with white British. The Chinese group in the UK has been associated with fewer health problems than other groups including lower mortality. ${ }^{20}$

The results of this study suggest that many ethnic groups are not receiving optimal primary care in terms of either access or quality. A recent review of ethnic inequalities in the UK reported persistent inequalities in health outcomes. ${ }^{19}$ While several policies aim to deliver equal access to healthcare, ethnic minorities are generally less satisfied with the care they receive than the White British population. The COVID-19 pandemic has renewed concerns that ethnic minorities are more likely to be infected and diagnosed with severe outcomes including death. ${ }^{21}$ Specifically, South Asian groups have been particularly hard hit in the second wave of the UK epidemic. Previous experience indicates that ethnic minorities are less likely to be vaccinated for a range of diseases and there are early indications that some ethnic groups might be more hesitant about getting vaccinated against COVID-19. ${ }^{22}$ This is also consistent with the findings of this study in terms of higher risk of emergency admission for vaccine-preventable diseases. ACSC indicators were introduced into the NHS Commissioning Outcome Framework in 2012. ${ }^{1}$ They are not direct indicators of healthcare delivery but are deemed to have value in the monitoring quality of care at a high level. Further studies would therefore be required to map out the more specific healthcare needs and healthcare accessibility for vulnerable groups. It will be important to identify health system factors as well as patient factors, for example, studying whether more can be done to increase access and quality of care. 


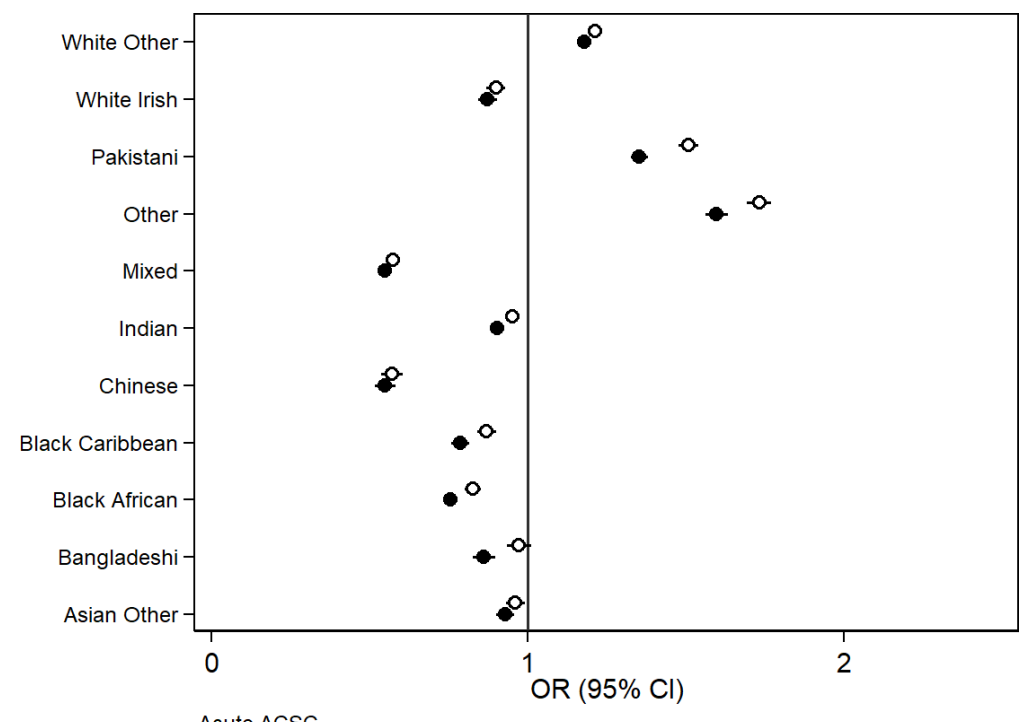

Acute ACSC

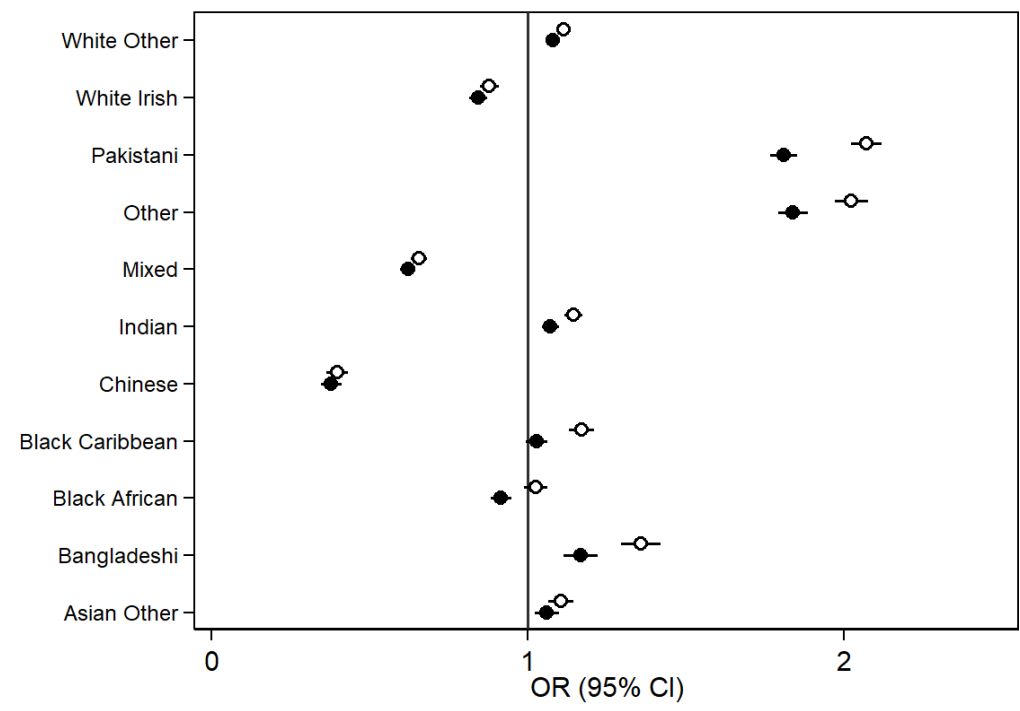

Chronic ACSC

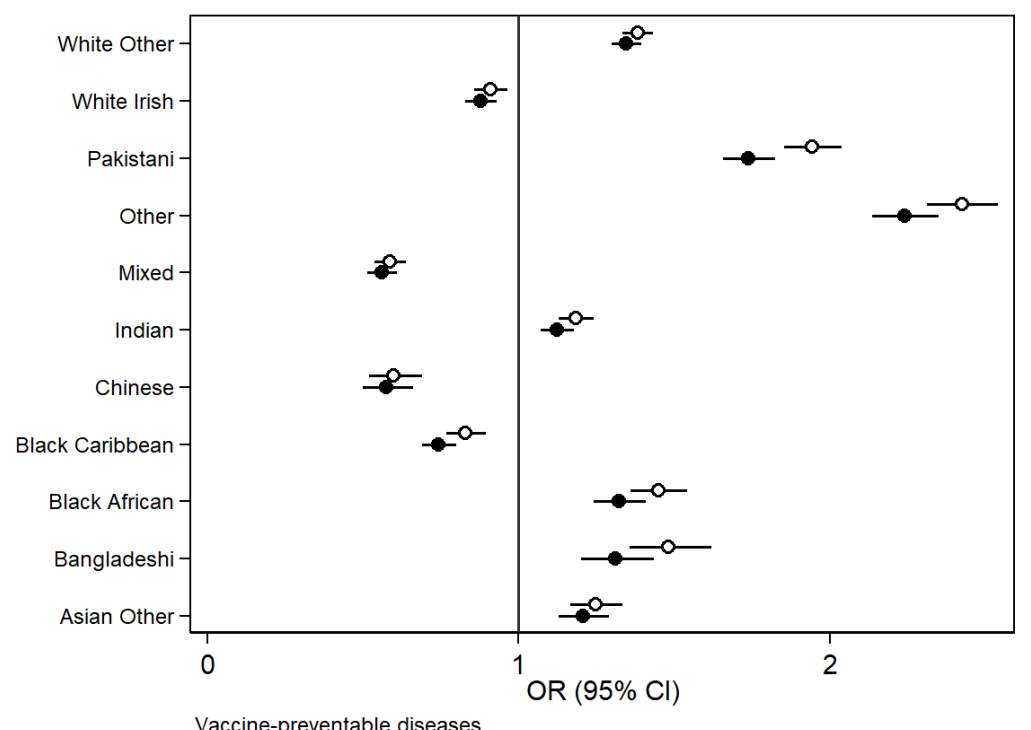

Figure 1 ORs of ACSC admission by ethnic group relative to white British adjusted for age, sex and area deprivation, 2011. Top: acute ACSC. Middle: chronic ACSC. Bottom: vaccine-preventable diseases. Open circles: risk adjusted for age group and sex. Filled circles: adjusted for age group, sex and area deprivation. ACSC, ambulatory care sensitive conditions. 
Table 3 Age-adjusted, sex-adjusted and deprivation-adjusted (OR $95 \% \mathrm{Cl}$ ) for the combined ACSC outcome

\begin{tabular}{lllll}
\hline Ethnic group & Age-sex-adjusted $\mathrm{OR}(95 \% \mathrm{Cl})$ & P value & Age-adjusted and sex-deprivation-adjusted OR $(95 \% \mathrm{Cl})$ & $\mathrm{P}$ value \\
\hline White British & Ref & - & Ref & - \\
Asian other & $1.04(1.02$ to 1.07$)$ & $<0.001$ & $1.01(0.98$ to 1.03$)$ & 0.640 \\
Bangladeshi & $1.13(1.10$ to 1.17$)$ & $<0.001$ & $0.99(0.96$ to 1.02$)$ & 0.590 \\
\hline Chinese & $0.51(0.49$ to 0.54$)$ & $<0.001$ & $0.49(0.47$ to 0.51$)$ & $<0.001$ \\
\hline Indian & $1.05(1.03$ to 1.07$)$ & $<0.001$ & $0.99(0.97$ to 1.01$)$ & 0.193 \\
\hline Pakistani & $1.74(1.71$ to 1.76$)$ & $<0.001$ & $1.54(1.52$ to 1.57$)$ & $<0.001$ \\
\hline Black African & $0.95(0.93$ to 0.97$)$ & $<0.001$ & $0.86(0.84$ to 0.88$)$ & $<0.001$ \\
\hline Black Caribbean & $0.98(0.96$ to 1.01$)$ & 0.173 & $0.88(0.86$ to 0.90$)$ & $<0.001$ \\
\hline Other & $1.92(1.88$ to 1.95$)$ & $<0.001$ & $1.76(1.73$ to 1.78$)$ & $<0.001$ \\
White other & $1.20(1.18$ to 1.21$)$ & $<0.001$ & $1.16(1.15$ to 1.18$)$ & $<0.001$ \\
White Irish & $0.88(0.86$ to 0.90$)$ & $<0.001$ & $0.85(0.83$ to 0.87$)$ & $<0.001$ \\
\hline Mixed & $0.60(0.59$ to 0.61$)$ & $<0.001$ & $0.57(0.56$ to 0.59$)$ & $<0.001$ \\
\hline
\end{tabular}

NHS-recorded ethnicity replaced with EE prediction where missing (Kandt and Longley, 2018). ${ }^{8}$

ACSC, ambulatory care sensitive conditions; EE, ethnicity estimator; NHS, National Health Service.

The maps showed regional effects in the incidence of preventable hospitalisation. Inner London and the old industrial centres of the Midlands and the North had higher incidence than the national average. These industrial and postindustrial areas are associated with persistent deprivation on multiple accounts ${ }^{1623-25}$ and an indication that preventable hospitalisation overlap with regional patterns of deprivation. The incidence for the Pakistani group was more widespread than for the White British and not only concentrated in the most deprived regions. The elevated risk of ACSC admission attenuated partly when adjusting for area deprivation, which again suggests that the difference between these groups and white British was only partly explained by residence in deprived areas.

The other ethnic group was associated with the highest risk of ACSC admissions. This is inherently not a well-defined group, which makes the interpretation of the results more complicated. It could highlight problems associated with a conglomerate of

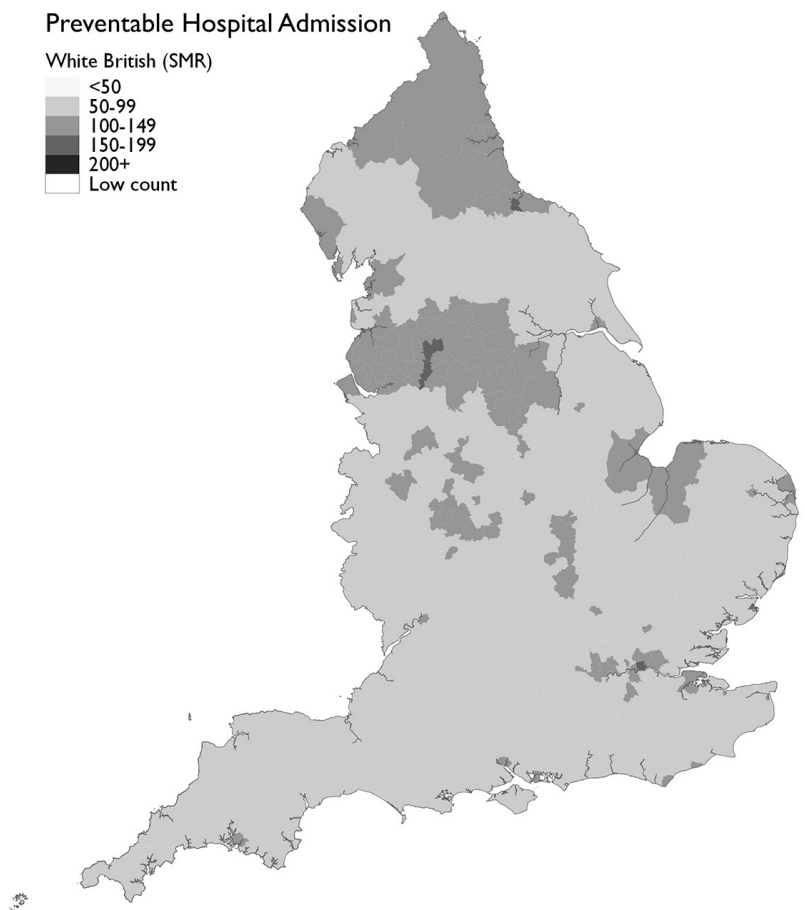

marginalised ethnic groups, but more detailed studies would be needed to unpack this.

\section{LIMITATIONS}

HES is a unique data set with both strengths and limitations. It is an administrative dataset that may also reflect time-variant healthcare system factors, for example, as a result of policy-driven target setting. As a particular strength, HES captures all hospital admissions commissioned by NHS England, which is estimated to cover 98\%-99\% of all hospital activity in England. ${ }^{26}$ Patients have recently gained the right to opt out of having their data used for research retrospectively. So far only a small proportion of HES patients have exercised this right. ${ }^{26}$ For our research, we rely on the accuracy of the coding of each episode of care and acknowledge that while the accuracy may vary, studies have found HES adequate for both research and managerial decision making. ${ }^{27}$

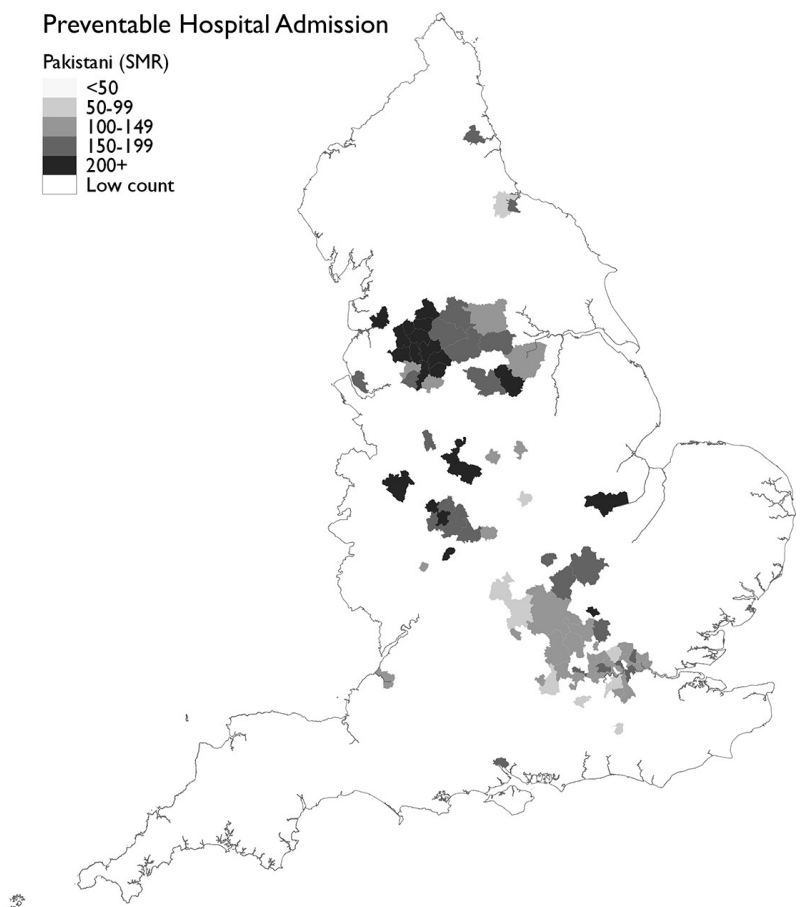

Figure 2 Preventable hospitalisation SMR by local authority district for white British and Pakistani in 2011. SMR, standardised morbidity ratio. 
Definitions for ACSC vary in the literature in the number of conditions that are included. We have aligned our definitions with those published by Bardsley et al, ${ }^{1}$ which are also used by the English NHS.

Recording of ethnicity will never be entirely objective as it relates to identity and data in this study were furthermore derived from two different sources of self-reported ethnicity, i.e. HES for admissions and Census 2011 for base population. It cannot be excluded from consideration that recorded ethnicity may vary with the timing, mode, and context of the response process. Moreover, the ethnicity data in HES is deemed to be self-reported, but in practice there will likely be instances in which it is staff-reported.

Recorded ethnicity information in HES was below 50\% before 2000 , but has since improved. ${ }^{728}$ Only $5.2 \%$ of patients in this 2011 study had missing ethnicity information. Combining HESrecorded ethnicity with surname derived EE, as in this study, will also not escape a degree of subjectivity, but is a way to develop a more complete analysis that avoids imputing as missing-atrandom. While the imputation increased the incidence for these groups in absolute terms, it only had a modest effect on relative risk and did not change the main findings of the study (figure 1, table 3, online supplemental figure S1 and table S2). Many bearers of Irish surnames today perceive themselves as White British. ${ }^{8} 12$ Surname imputation is more error prone as a consequence. Similar results were nonetheless obtained with and without surname imputation for this group too.

Mixed ethnic group could not be enhanced in the analyses as there is no category for it in the current version of the EE

\section{What is already known on this subject}

- Emergency hospital admissions are distressing for patients, associated with poorer long-term outcomes and are costly to the healthcare system.

- Indicators for admissions considered preventable have been defined and known as ambulatory care sensitive conditions (ACSC). ACSC admissions have been associated with patients under the age 5 years, the elderly, deprivation and ethnicity. The English National Health Service monitors ACSC in the general population and saw a 40\% rise between 2001 and 2011.

- Studies in USA, New Zealand and Scotland have found higher risk of ACSC admission for many ethnic minorities compared to the white majority populations.

\section{What this study adds}

- This is the first study of its kind in England and the first investigation of the different ambulatory care sensitive conditions (ACSC) outcomes for all major ethnic groups in the UK.

- Preventable emergency admissions ACSCs were especially high among patients with Bangladeshi, Pakistani, black African, white other or other background with up to twofold differences compared to the white British majority group in England.

- The results for the different ACSC outcomes suggest that the identified ethnic groups may not be receiving optimal primary care. software. About $2 \%$ of the population identified as mixed

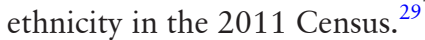

The deprivation adjustment of the regression analyses was conducted at the level of local authorities because of data availability. It is possible that more of the variation could have been explained if it had been possible to adjust at a finer level of geography.

\section{CONCLUSIONS}

Preventable emergency admissions were especially high among patients with Bangladeshi, Pakistani, black African, white other or other background with up to twofold differences compared with the white British majority group. Further studies will be needed to uncover specific barriers. Greater vigilance among health staff along with outreach activities to increase uptake of health checks and other interventions could potentially increase early detection of chronic conditions in these groups. Problems with acute conditions suggest underuse of general practices and community services. For vaccine-preventable diseases, ethnic disparities suggest that the offer of vaccinations in high-risk groups such as the elderly does not reach all ethnic groups equally well. There was some geographical overlap for the combined ACSC endpoint with regions of high levels of deprivation, but the geographical distribution was more dispersed for the Pakistani compared with the white British group. The inequalities in ACSC revealed in this study suggest that several ethnic minorities are not receiving optimal primary and preventive care.

Correction notice This article has been corrected since it first published. The open access licence type has been changed to CC BY.

Contributors All authors contributed to the planning and reporting of this work and the responses to reviewers (JP, JK and PL). JP conducted the analysis of the data. JK designed and arranged the ethnicity coding of HES records. The corresponding author attests that all listed authors meet the authorship criteria and that no others meeting the criteria have been omitted.

Funding The UK Economic and Social Research Council is acknowledged for its support for the UCL Consumer Data Research Centre (CDRC) enabling this research (Grant ES/L011840/1)

Disclaimer The funder had no direct role in relation to the specific study.

Map disclaimer The depiction of boundaries on this map does not imply the expression of any opinion whatsoever on the part of BMJ (or any member of its group) concerning the legal status of any country, territory, jurisdiction or area or of its authorities. This map is provided without any warranty of any kind, either express or implied.

Competing interests None declared.

Patient consent for publication Not required.

Ethics approval Ethical approval was obtained from Bromley REC (Reference: 13/ LO/1355) for analyses of patient-level HES data. The HES data licence reference is DARS-NIC-28051-Q3K7L.

Provenance and peer review Not commissioned; externally peer reviewed

Data availability statement Data may be obtained from a third party and are not publicly available. The data that support the findings of this study are available from NHS Digital, but restrictions apply to the availability of these data, which were used under license for the current study, and so are not publicly available.

Supplemental material This content has been supplied by the author(s). It has not been vetted by BMJ Publishing Group Limited (BMJ) and may not have been peer-reviewed. Any opinions or recommendations discussed are solely those of the author(s) and are not endorsed by BMJ. BMJ disclaims all liability and responsibility arising from any reliance placed on the content. Where the content includes any translated material, BMJ does not warrant the accuracy and reliability of the translations (including but not limited to local regulations, clinical guidelines, terminology, drug names and drug dosages), and is not responsible for any error and/or omissions arising from translation and adaptation or otherwise.

Open access This is an open access article distributed in accordance with the Creative Commons Attribution 4.0 Unported (CC BY 4.0) license, which permits others to copy, redistribute, remix, transform and build upon this work for any 
purpose, provided the original work is properly cited, a link to the licence is given, and indication of whether changes were made. See: https://creativecommons.org/ licenses/by/4.0/.

\section{ORCID iD}

Jakob Petersen http://orcid.org/0000-0002-6659-7028

\section{REFERENCES}

1 Bardsley M, Blunt I, Davies S, et al. Is secondary preventive care improving? Observational study of 10 -year trends in emergency admissions for conditions amenable to ambulatory care. BMJ Open 2013;3. doi:10.1136/ bmjopen-2012-002007. [Epub ahead of print: 02 Jan 2013].

2 Busby J, Purdy S, Hollingworth W. How do population, general practice and hospital factors influence ambulatory care sensitive admissions: a cross sectional study. BMC Fam Pract 2017;18:67

3 Hodgson K, Deeny SR, Steventon A. Ambulatory care-sensitive conditions: their potential uses and limitations. BMJ Qual Saf 2019;28:429-33.

4 Katikireddi SV, Cezard G, Bhopal RS, et al. Assessment of health care, hospital admissions, and mortality by ethnicity: population-based cohort study of healthsystem performance in Scotland. Lancet Public Health 2018;3:e226-36.

5 Blunt I. Focus on preventable admissions, 2013. Available: https://www.health.org.uk/ publications/qualitywatch-focus-on-preventable-admissions [Accessed 16 Jul 2020].

6 Dalla Zuanna T, Spadea T, Milana M, et al. Avoidable hospitalization among migrants and ethnic minority groups: a systematic review. Eur J Public Health 2017;27:861-8.

7 Mathur R, Bhaskaran K, Chaturvedi N, et al. Completeness and usability of ethnicity data in UK-based primary care and hospital databases. J Public Health 2014;36:684-92.

8 Kandt J, Longley PA. Ethnicity estimation using family naming practices. PLoS One 2018;13:e0201774.

9 WHO. ICD-10 Version:2016, 2016. Available: https://icd.who.int/browse10/2016/en [Accessed 17 Jan 2020].

10 Office for National Statistics. Ethnic group, National identity and religion, 2011. Available: https://www.ons.gov.uk/methodology/classificationsandstandards/ measuringequality/ethnicgroupnationalidentityandreligion [Accessed 4 Apr 2021].

11 NHS. Monitoring equality and health inequalities: a position paper. Leeds, UK Equality and Health Inequalities Team; 2015. https://www.england.nhs.uk/wp-content/ uploads/2015/03/monitrg-ehi-pos-paper.pdf [Accessed 4 Jun 2021].

12 Petersen J, Kandt J, Longley PA. Names-based ethnicity enhancement of hospital admissions in England, 1999-2013. Int J Med Inform 2021;149:104437.
13 Kandt J, van Dijk J, Longley PA. Family name origins and intergenerational demographic change in Great Britain. Ann Am Assoc Geogr 2020;110:1726-42.

14 Mateos P, Longley PA, O'Sullivan D. Ethnicity and population structure in personal naming networks. PLoS One 2011;6:e22943.

15 Lakha F, Gorman DR, Mateos P. Name analysis to classify populations by ethnicity in public health: validation of Onomap in Scotland. Public Health 2011;125:688-96.

16 Department for Communities and Local Government. The English indices of deprivation 2015 Stat Release; 2015: 38.

17 StataCorp. Stata statistical software: release 14. Texas, USA: College Station, 2015.

18 QGIS. Quantum GIS (QGIS), 2021. Available: http://www.qgis.org/en/site/ [Accessed 15 Apr 2021].

19 Chouhan K, Nazroo J. Health inequalities. In: Ethnicity, Race and Inequality in the UK State of the Nation. Bristol, UK: Policy Press, 2020: 73-92.

20 Bhopal RS, Gruer L, Cezard G, et al. Mortality, ethnicity, and country of birth on a national scale, 2001-2013: a retrospective cohort (Scottish health and ethnicity linkage study). PLoS Med 2018;15:e1002515.

21 Mathur R, Rentsch CT, Morton CE, et al. Ethnic differences in SARS-CoV-2 infection and COVID-19-related hospitalisation, intensive care unit admission, and death in 17 million adults in England: an observational cohort study using the OpenSAFELY platform. Lancet 2021;397:1711-24.

22 Scientific Advisory Group for Emergencies. Factors influencing COVID-19 vaccine uptake among minority ethnic groups, 2020. Available: https://www.gov.uk/ government/publications/factors-influencing-covid-19-vaccine-uptake-amongminority-ethnic-groups-17-december-2020 [Accessed 17 May 2021].

23 Cummins S, Curtis S, Diez-Roux AV, et al. Understanding and representing 'place' in health research: A relational approach. Soc Sci Med 2007;65:1825-38.

24 Darlington-Pollock F, Norman P. Establishing a framework of analysis for selective sorting and changing health gradients. Popul Space Place 2020:e2359.

25 Steel N, Ford JA, Newton JN, et al. Changes in health in the countries of the UK and 150 English local authority areas 1990-2016: a systematic analysis for the global burden of disease study 2016. The Lancet 2018;392:1647-61.

26 Herbert A, Wijlaars L, Zylbersztejn A, et al. Data resource profile: Hospital episode statistics admitted patient care (Hes APC). Int J Epidemiol 2017;46:1093-1093i.

27 Burns EM, Rigby E, Mamidanna R, et al. Systematic review of discharge coding accuracy. J Public Health 2012;34:138-48.

28 Smith L, Norman P, Kapetanstrataki M, et al. Comparison of ethnic group classification using naming analysis and routinely collected data: application to cancer incidence trends in children and young people. BMJ Open 2017;7:e016332.

29 Office for National Statistics. 2011 census: aggregate data (England and Wales). Available: http://www.nationalarchives.gov.uk/doc/open-government-licence/ version/2 [Accessed 27 Feb 2015]. 